\title{
Dealing with high gas loads in uhv systems: some recent developments at CERN (LEAR)
}

\author{
A M Poncet, CERN, PS Division, 1211 Geneva 23, Switzerland
}

\begin{abstract}
Particle storage rings and accelerators with uhv systems providing pressures less that $10^{-11} \mathrm{mbar}$ are or have been conceived with now well-established standards and technologies. Among other things, a key to success is the careful choice of materials and treatments to reduce the outgassing. The growing interest in low energy particle storage rings equipped with exotic and high gas load equipment such as electron coolers and internal gas jet targets has triggered the search for new solutions in uhv techniques. Some recent developments around the CERN Low Energy Antiproton Ring (LEAR) involve the design of new pumps using low temperature nonevaporable getters (NEG). These are comparable in pumping speed performance with titanium sublimation pumps, but have a much increased pressure and capacity range. The design principles, Monte Carlo calculation methods, and preliminary performance are presented.
\end{abstract}

\section{Introduction}

The LEAR storage ring at CERN has now been operated successfully for almost $4 \mathrm{yr}$ with an average residual pressure in the low $10^{-12}$ mbar range ${ }^{1}$. The uhv system of this high energy physics tool was built using the most up to date techniques and technologies that had been developed or applied at the now dismantled proton storage ring ISR $^{2}$. In particular, strict choice of materials, heat treatments, cleaning procedures and an elaborate bakeout system have reliably reduced the overall outgassing rates to the $10^{-13} \mathrm{mbar} 1 \mathrm{sec}^{-1} \mathrm{~cm}^{-2}$ range with negligible leak occurrence. With these very low gas loads, the pumping task can be most economically and efficiently ensured by condensed titanium layers (viz titanium sublimation pumps) for active gases, while sputter ion pumps take care of less readily pumpable gases such as methane and argon. After each exposure to air, the sublimation pumps are usually flashed 10-15 times during pumpdown and after bakeout, thus providing a few tens of condensed titanium monolayers, after which they do not need to be operated for periods of months because at the nominal operating pressure the gas load is so small that the saturation rate is negligible.

With the advent of new, more sophisticated machine and physics equipment such as electron coolers ${ }^{3}$ and gas jet targets ${ }^{4}$ which, in operation, provide large gas loads and virtually no space for pumping, the task of providing very low pressures becomes extremely difficult ${ }^{5}$ because all the 'classical' solutions have drawbacks. High gas throughputs can be obtained with getters such as sublimated titanium or non-evaporable getters at the expense of continuous operation at elevated temperature using large amounts of electrical power, thus heating which may be difficult to remove in the space available and which is usually detrimental for the required low base pressure. Extremely low limit pressure condensation cyropumps have also been applied to the high gas throughput problem ${ }^{12}$. However, there are cases where their bulky size, cryogenic feeds and expensive operation cannot be adopted easily.

\section{Non-evaporable getters (NEG)}

NEG pumps using commercially available getters such as ST 101 (konstantan strip onto which is bonded a powder mixture of $\mathrm{Zr}$ $84 \%-\mathrm{Al} 16 \%$ manufactured by SAES Getters, Milano, Italy) have already been used successfully in the uhv system of devices having abnormally high gas loads with very little space for pumping ${ }^{5}$. However, in reliably applying the high electrical power required for activation of the getter modules, technical difficulties have been encountered which make the operation possible, but delicate.

With the appearance of getters which can be activated at temperatures close to those which are normally used for bakeout of uhv systems (viz $300-450^{\circ} \mathrm{C}$ ), providing good sorption characteristics when operated at room temperature after their initial activation, the inconvenience of supplying and dissipating a large amount of electrical power disappears. In addition the external heating system normally used for in situ bakeout, if dimensioned properly, can be used for activation during the normal pump down sequence, thus avoiding the need for high current electrical feedthroughs.

Such a low temperature $\left(450^{\circ} \mathrm{C}\right)$ getter called ST 707 , available in the form of continuous konstantan ribbon on the faces of which is sintered a powdered mixture of $\mathrm{Zr} / \mathrm{Zr}-\mathrm{V}-\mathrm{Fe}$ alloy, is also manufactured by SAES Getters ${ }^{6}$. The physical and pumping characteristics such as specific gas sticking coefficients and capacities, equilibrium pressures for various operating temperatures and conditionings have been published in the literature ${ }^{6,7}$. Some indicative data is summarized in Table 1 and compared to corresponding values obtained with a freshly evaporated titanium film ${ }^{8}$ for two common uhv gases, at uhv pressures. 


\begin{tabular}{lllll}
\hline Table 1 & & & \\
\hline (Room & \multicolumn{3}{l}{ Sticking probability } & \multicolumn{2}{l}{ Capacity $\left(\mathrm{mbar} \mathrm{l} \mathrm{cm}^{-2}\right)$} \\
temperature) & ST 707 & $\mathrm{Ti}$ & $\mathrm{ST} 707$ & $\mathrm{Ti}$ \\
\hline $\mathrm{H}_{2}$ & 0.007 & 0.05 & 0.1 & 0.0002 \\
$\mathrm{CO}$ & 0.01 & 0.5 & 0.001 & 0.0002 \\
\hline
\end{tabular}

The specific pumping speed at room temperature of ST 707 activated at $450^{\circ} \mathrm{C}$ for $10 \mathrm{~min}$ under uhv conditions is lower-and significantly for $\mathrm{CO}$ than that of a fresh titanium layer. On the other hand, the higher capacity for ST 707 due to a large porosity and effective-to-nominal surface ratio should allow a higher gas throughput at room temperature. However, in uhv systems with abnormally high gas loads and little space for pumping-which is the case of interest-large amounts of getter strip may be required in a small volume if for practical reasons one cannot perform frequent reconditioning. This operation conducted at an intermediate temperature is aimed at restoring the sorption ability through bulk diffusion of the previously sorbed monolayer.

In addition to high gas throughput (i.e. large pumping speed and capacity), another interesting feature which should govern the design of a pump based on the use of ST 707, should be its ability to provide large pressure gradients so as to confine unavoidable high pressure zones in the immediate vicinity of the gas load (high power electron guns and collectors used in coolers, internal gas jet targets, etc.).

\section{A possible pump arrangement}

A prototype pump based on ST 707 has been built and is shown in the schematic drawing in Figure 1. The getter strip is wrapped like an accordion, folded in the form of doughnuts which are then piled inside a stainless steel cylinder. The hole in the centre leaves passage for beams in accelerator applications and pumping is provided exactly where needed. In practice, the pump can be shaped to fit almost any boundary conditions imposed by beam size, available length and maximum outer diameter. The getter occupies the full volume, with little lost space except externally for bakeout jackets and is hit by the molecules to be pumped under very favourable conditions. Indeed, incoming molecules always hit the getter at grazing angle thus limiting direct reflexion, and upon bouncing off the getter, see minimum getter-free solid angle.

Obviously there are many free parameters that one can try to optimize in the design of such a pump: length; height and number of folds; outer diameter; exact fold gcometry; activation and regeneration procedures in uhv conditions. However, optimization can also be dictated by freezing some dimensions due to the peculiar environment. The analysis and experiment described

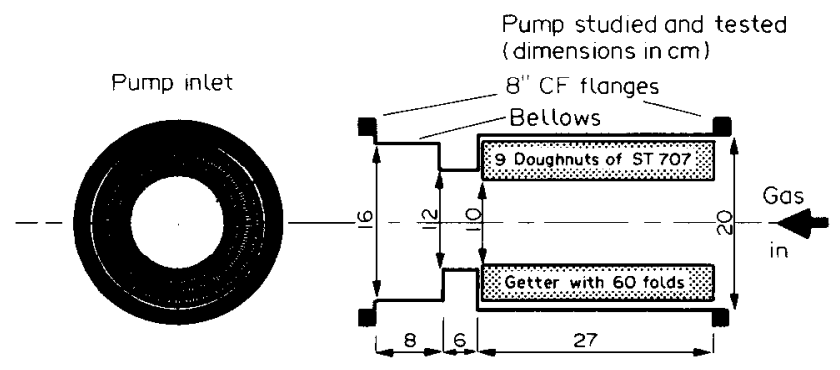

Figure 1. Schematic diagram of non-evaporable getter pump. below was done with specific future applications in mind for LEAR and does not pretend to be complete. In particular, overall length, inner and outer diameters (i.e. fold height) are more or less fixed and given by the beam and machine geometry. Therefore, the main parameter which has been considered is the number of folds, which for a given volume gives the length-i.e. price- of getter to be used.

\section{Monte Carlo calculations of transmission and adsorption probabilities}

A general purpose molecular tracking program "CARLO', developed on a HP9845B desktop computer, has been used to study transmission, adsorption and rejection probabilities for various pump configurations. The program, using the well-known Monte Carlo technique ${ }^{10}$, traces molecular trajectories in Cartesian coordinates through consecutive tubular segments which can be filled with two types of structures: axisymmetric baffles and azimuthally wrapped ribbons. Wall sorption is included via the so called sticking coefficient (range 0-1), defined as the probability that a molecule is permanently adsorbed upon a collision with a surface. The program treats steady flows under molecular conditions. The particles enter the structure by molecular effusion
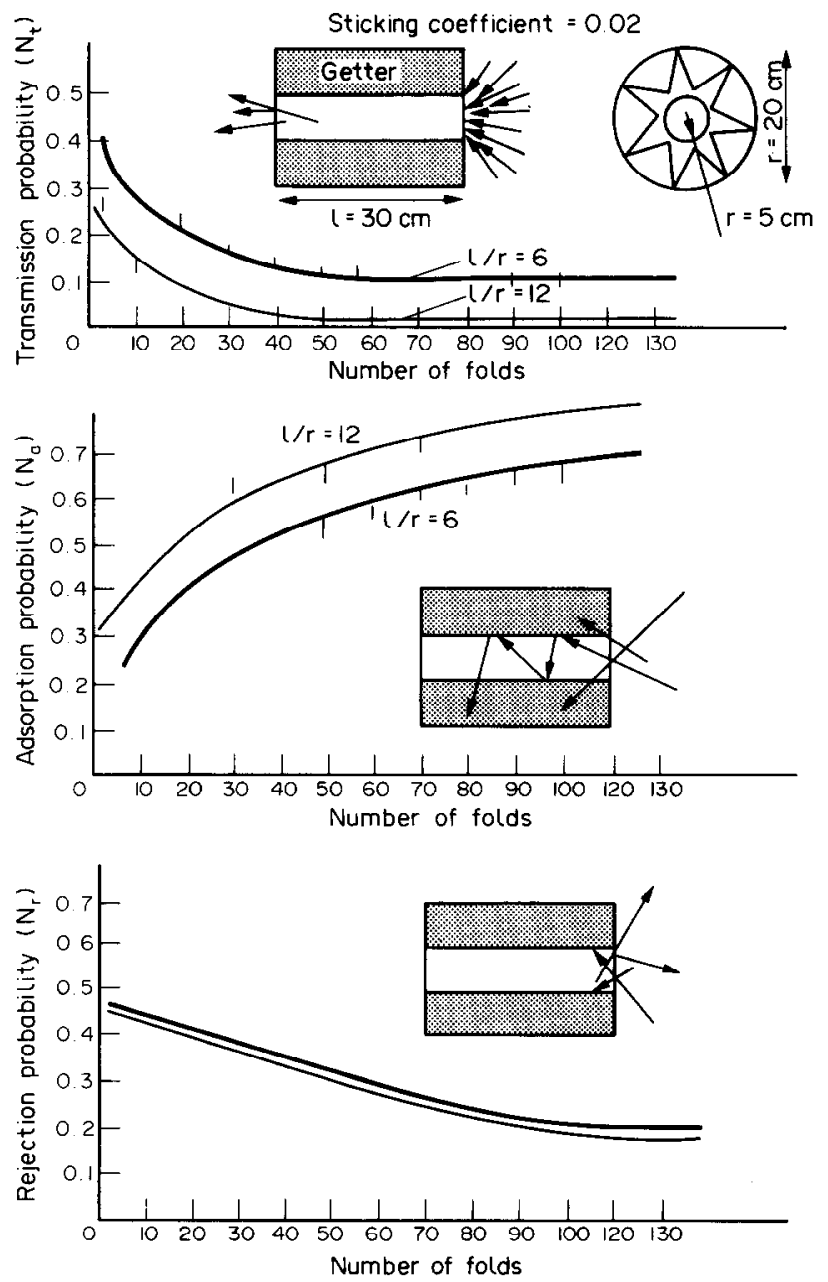

Figure 2a. Monte Carlo calculations of transmission, adsorption and rejection probabilities as a function of the number of folds $(\mathrm{St}=0.02)$ for two inner tube radius to length aspect ratios $(l / r=6$ and $l / r=12)$. 
from a large chamber and reflexion on surfaces is diffuse; the statistical distribution of the direction of propagation around the normal to the surface follows the cosine law. The standard deviation around the calculated values, inversely proportional to the square root of the sample size $(1000-5000$ molecules in this report's calculations), usually ranges from less than one to a few per cent.

Figure $2 \mathrm{a}$ shows transmission, adsorption and rejection probabilities as a function of the number of getter folds for the pumping part of the arrangement described above $(l / r=6)$, for a realistic sticking coefficient of 0.02 . Also shown are the results of calculations for a twice as large length to inner radius ratio of $l / r=12$. It is seen that in our case of interest $(l / r=6)$ there is little gain to be expected in transmission probability beyond about 60 folds. Also, doubling the number of folds from 60 only results in about $15 \%$ increase in adsorption probability, i.e. pumping speed. Therefore, 60 folds were chosen for the first prototype tests. Also apparent is the interest of increasing the length of the tube equipped with getter, at equal developed length: an $l / r=12$ configuration will have the same pumping speed with only 30 folds as an $l / r=6$ tube with 60 folds, but the transmission probability will be 5 times lower. For the two cases studied, the rejection probability is roughly identical: one can expect the rejection probability to be only sizeable at the pump entrance.

The dependence of transmission, adsorption and rejection probabilities on the value of the sticking coefficient has been calculated and compared with those of a smooth tube of identical inner dimensions. This is shown in Figure $2 b$, where it is clear that optimum operating conditions would require a sticking coeffi-
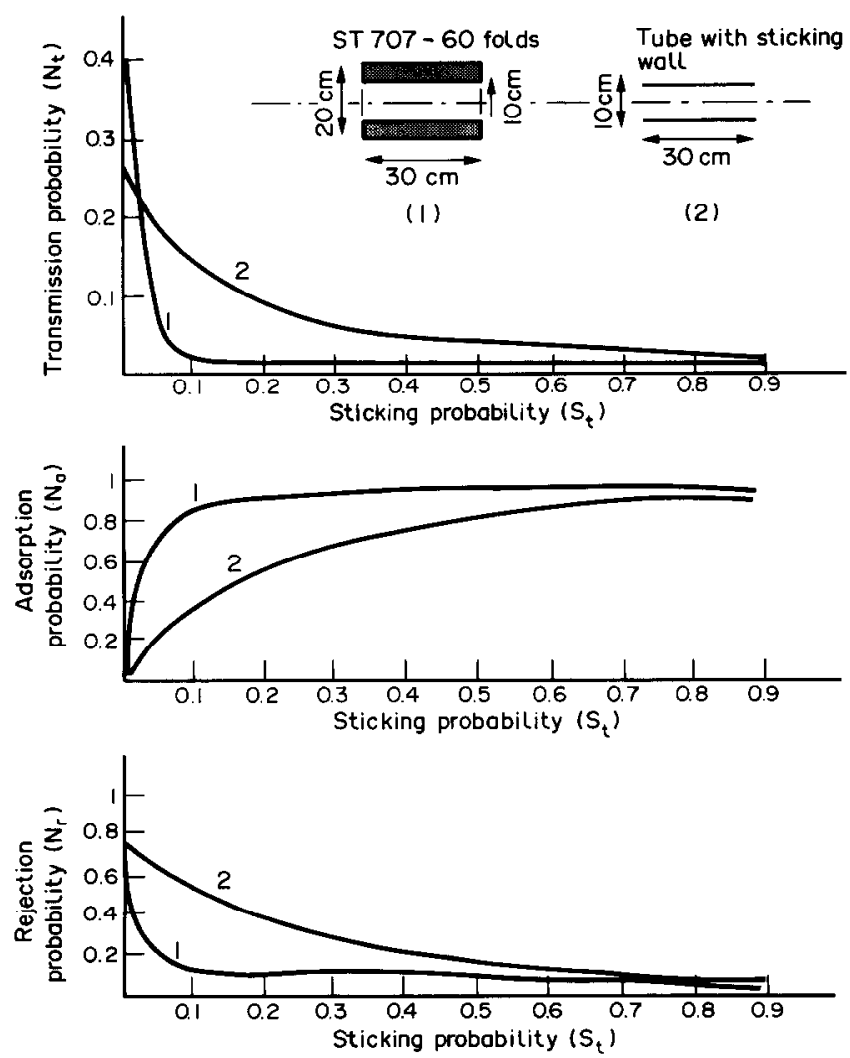

Figure 2b. Sume calculations as function of the sticking coefficient, for a tube woth 60 getter folds and $l / r=6$. Curve 2 is for a smooth tube of identical length to radius ratio, with sticking wall. cient of 0.1 , which is $3-5$ times higher than is achievable with activations at temperatures of $450^{\circ} \mathrm{C}$.

However, comparison with a smooth tube shows that at equal probabilities a sticking coefficient of 0.02 on the getter is roughly equivalent to 0.2 for the smooth tube. In other words, wrapping the getter in accordion shape provides an apparent sticking coefficient of 0.2 , a ten fold gain in efficiency with respect to plain ribbon.

\section{Pumping speed measurements}

The pumping speed of the prototype pump described above has been measured for $\mathrm{H}_{2}$ and $\mathrm{CO}$ with a Fischer-Momsen test dome ${ }^{11}$, under uhv conditions. This dome was equipped with modulated Bayard-Alpert ion gauges, a residual gas analyser and a $201 \mathrm{~s}^{-1}$ sputter ion pump for chemically inactive gases. The pump and the dome equipped with their bakeout jackets were baked at $300^{\circ} \mathrm{C}$ for $24 \mathrm{~h}$, while being evacuated with a turbomolecular pump. Towards the end of the period at $300^{\circ} \mathrm{C}$, the pressure in the test dome being in the $10^{-8}-10^{-9} \mathrm{mbar}$ range, the power on the pump heating jacket was raised to achieve a temperature of $340^{\circ} \mathrm{C}$, measured with a thermocouple placed on the getter, for a period of $1 \mathrm{~h}$. The pumping speed for $\mathrm{CO}$ was then measured at room temperature as a function of the gas load, with throughputs of 2.5-8 $10^{-6}$ mbar $1 \mathrm{~s}^{-1}$. After saturation and without letting the system up to air, the activation was repeated at higher temperature $\left(420^{\circ} \mathrm{C}-475 \mathrm{~W}\right.$ on pump housing $)$ at the end of a full bake and the pumping speed measured again under the same conditions. The two subsequent measurements were performed without bakeouts, solely after reconditionings of the pump at $220^{\circ} \mathrm{C}$ for $1 \mathrm{~h}$.

The results are shown on Figure 2c. While the initial pumping speeds are 1500 and $2000 \mathrm{~s}^{-1}$ for $\mathrm{CO}$ and $\mathrm{H}_{2}$, they became after a 10 mbar 1 saturation respectively 400 and $1500 \mathrm{l} \mathrm{s}^{-1}$.

Comparison with theoretical pumping speed values as a function of sticking coefficients shown on Figure 2d, as calculated with our Monte Carlo program, indicates that the best achieved sticking coefficient for $\mathrm{CO}$ should be initially $0.02-0.03$. The pump ultimate pressure was consistently measured in the $2-3 \times 10^{-11}$ mbar range, with mostly hydrogen as residual gas, throughout the testing period and after an $\mathrm{H}_{2}$ saturation of more than 50 mbar 1 . This confirms previous observations of ST $707 \mathrm{H} 2$ equilibrium pressure at room temperature?
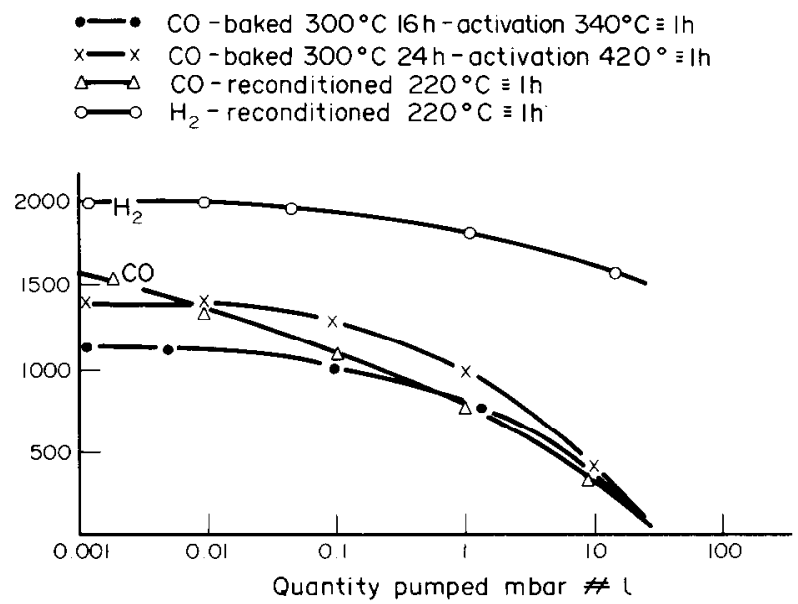

Figure 2c. Pumping speed $\left(1 \mathrm{~s}^{-1}\right)$ at room temperature (gas load: $2.5-8 \times 10^{-6}$ mbar l s$^{-1}$ ). 


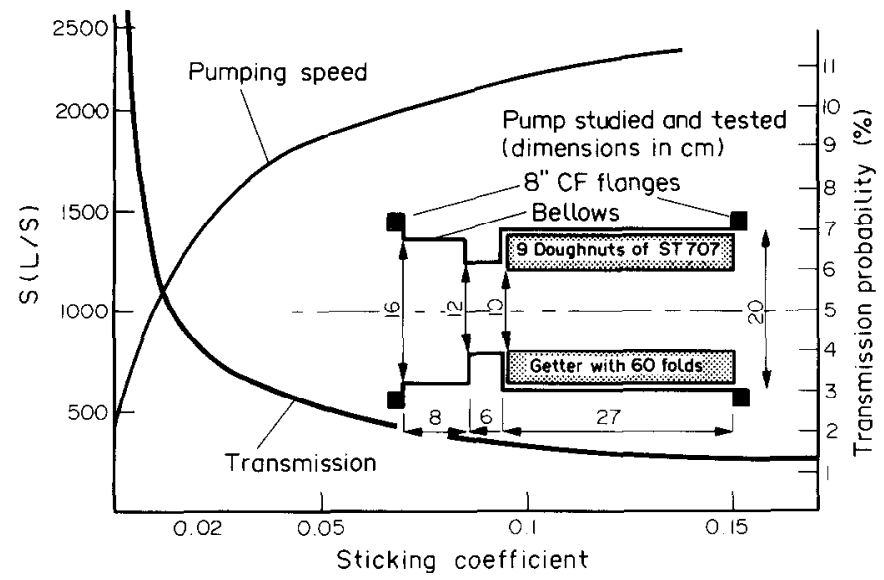

Figure 2d. Theoretical pumping speed for $\mathrm{CO}$ as a function of the sticking coefficient.

\section{Conclusion and further studies}

Although preliminary, this work shows the applicability of the ST 707 getter ribbon in uhv systems with high gas loads and limited space for pumping. Indeed the gas throughput capability of the arrangement described in this report is $2-3$ orders of magnitude larger than that of a titanium sublimation pump operating at room temperature, for the same bulk size. It compares favourably with sputter ion pumps as far as throughput, with a much larger pumping speed for active gases at low pressures and at identical size. The pump can be custom built to fit any particular environment, and its performance predicted, a non-negligible advantage at the design stage. Although requiring further study its operation is simple--needing activation and recondition- ing - and should not pose any problem in densely packed environments due to the low power required. Of course, there is room for further studies and optimization, for example, shaping of the folds or introduction of intermediate baffles and this is what we plan to do with future applications in LEAR in mind.

\section{Acknowledgements}

A large part of the work on the Monte Carlo program and pumping speed measurements has been performed by Johan Bjon, a visitor from the Forskningsinstitutet for Atomfysik (AFI) of Stockholm. Michel Brouet and Marc Girardini (CERN) have participated in the design, construction and testing of the pump.

\section{References}

${ }^{1}$ M Brouet et al, The Ultra High Vacuum System for the Low Energy Antiproton Ring (LEAR). Design, Cost and Performances, CERN/PSML/83-42 (1983).

${ }^{2}$ E Fischer, J Vac Sci Technol, 9, 1203 (1972).

${ }^{3} \mathrm{H}$ Haseroth et al, The LEAR Electron Cooler, CERN/PS/84-28 (1984).

${ }^{4} M$ Macri, CAS, Antiprotons for Colliding Beam Facilities, p 469, CERN 84-15 (1984).

${ }^{5} \mathrm{M}$ Brouet et al, An Ultra High Vacuum System for Coolers, CERN/PS/85-4 (1985).

${ }^{6}$ B Ferrario et al, A New Generation of Porous Non-Evaporable Getters, SAES Getters SpA, Via Gallarate 215,20151, Milan, Italy.

${ }^{7} \mathrm{H}$ C Hseuh et al, J Vac Sci Technol, A1, 1283 (1983).

${ }^{8}$ P A Redhead et al, The Physical Basis of Ultra High Vacuum, Chapman \& Hall, London (1968).

9 A M Poncet, CARLO: a molecular tracking program, CERN PS Internal Note (to be published).

${ }^{10} \mathrm{C}$ G Smith et al, $J$ Vac Sci Technol, 3, 92 (1966)

${ }^{11}$ E Fischer et al, Vacuum, 17, 309 (1967).

427 (1979).

${ }^{12}$ C Benvenuti, Vacuum, 29, 